\title{
Adaptation of the Standard 12-Lead ECG System Focusing on Atrial Electrical Activity
}

\author{
Z Ihara $^{1}$, V Jacquemet ${ }^{1}$, J-M Vesin ${ }^{1}$, A van Oosterom $^{2}$ \\ ${ }^{1}$ Swiss Federal Institute of Technology Lausanne, Switzerland \\ ${ }^{2}$ Lausanne University Hospital Center, Switzerland
}

\begin{abstract}
An adaptation is presented of the positioning of some of the electrodes of the standard 12-lead ECG, aimed at extracting more information on atrial activity. It uses new positions for four of the six precordial electrodes, anchored to the remaining two. Its performance was tested by applying it to ECG signals during atrial fibrillation, simulated by means of a biophysical model of human atria and thorax. This enabled the analysis to be carried out without the interference of the ventricular activity. The signals simulated on each of the two lead systems, denoted here as ECG and ACG (atriocardiogram), were compared by studying the singular values of the data matrix $($ size $9 \times N)$ representing the independent information in the data. After normalization with respect to the first singular value, the singular value spectrum of the ACG lied well above that of the ECG data. The results indicate that the ACG lead system provides more information on the atrial electric activity than the standard 12-lead ECG configuration.
\end{abstract}

\section{Introduction}

The standard 12-lead ECG is the most commonly used tool for the non-invasive diagnostics of cardiac arrhythmias. The information content of atrial electrical activity in the signals recorded at the conventional lead positions may be limited due to the historical emphasis of the interest in ventricular activity. The current interest in the diagnosis of atrial fibrillation (AF) prompted our search for improved lead systems.

The ultimate introduction of any adapted lead system in a clinical routine will be facilitated if it extends, rather than replaces, the current clinical routine. It is for this reason that the adapted lead system (the ACG lead system) was anchored to those of the current practice. Of the nine electrodes involved in recording the standard 12-lead ECG, the two precordial electrodes lying closest to the atria, $\mathrm{V}_{1}$ and $\mathrm{V}_{2}$, were included in the ACG system, as were the limb electrodes $V_{R}, V_{L}$ and $V_{F}$. Since the 12-lead ECG con- tains at most 8 independent signal components, the ACG extracts at most half of its information from the new positions. This contribution reports on the usefulness of the ACG configuration when tested on simulated AF signals.

During the entire cardiac cycle, there is only a limited time window available for observing the atrial activity during AF in a manner that is uncontaminated by the presence of the far greater contributions of ventricular electric activity: the QRST complexes. This clearly hampers the optimization of lead systems on the basis of observed body surface potentials. As an alternative, this study uses a biophysical model of the genesis of AF signals that has been developed. The model includes as its major elements descriptions of the active electric sources during AF, as well as their expression on the thorax.

\section{Materials and Methods}

\subsection{Simulation of $\mathbf{A F}$}

Body surface potentials of AF were simulated on biophysical models of human atria and thorax, whose geometries are built by MRI slices taken on healthy subjects and discretized in meshed surfaces.

The transmembrane potentials during AF were computed on the $3 \mathrm{D}$ model, using a reaction-diffusion system [1-3] based on the ion kinetics described by Courtemanche et al. [4]. Eight AF episodes were simulated with the atrial model containing inhomogeneities in action potential duration [1]. The episodes were initiated by applying rapid pacing at different sites in the right and the left atrium.

The electric activity was then expressed on the closed outer surface of the atrial model as the strengths of the equivalent double layer (EDL) source elements [1]. The transfer of the EDL source to the body surface was calculated by means of the boundary element method (BEM) [5], including the inhomogeneous conduction properties of the model of the thorax [5-7]. The resulting body surface potentials $\boldsymbol{\Phi}$ on the thorax model were documented at its 300 vertices, of which the ECG and ACG lead positions are subsets. The signals were generated with a time reso- 

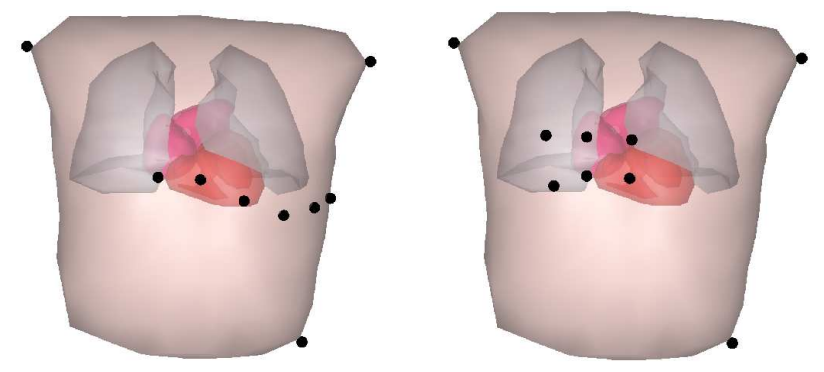

Figure 1. Display of the biophysical model and the lead systems used. The thorax includes the atria, ventricles and the lungs. The black dots indicate the electrode positions of the lead systems. Left, the standard 12-lead ECG and right, the ACG.

lution of $1 \mathrm{~ms}$. The eight simulated AF episodes were used as test signals for the evaluation of the lead systems.

\subsection{Evaluation}

The proposed ACG leads also uses nine electrodes, five among which $\left(\mathrm{V}_{\mathrm{R}}, \mathrm{V}_{\mathrm{L}}, \mathrm{V}_{\mathrm{F}}, \mathrm{V}_{1}\right.$ and $\left.\mathrm{V}_{2}\right)$ are anchored to the standard positions while the remaining four $\left(\mathrm{V}_{3}\right.$ to $\left.\mathrm{V}_{6}\right)$ are rearranged. The precordial electrodes are aligned so as to form a $2 \times 3$ grid on the upper right chest, lying over the atria: successively, electrode $V_{3}$ is repositioned one intercostal space above $\mathrm{V}_{2}$, similarly $\mathrm{V}_{4}$ above $\mathrm{V}_{1}, \mathrm{~V}_{5}$ to the right of the new $V_{4}$ position, and $V_{6}$ below the new $V_{5}$ position. The configuration is shown on the right of Figure 1. Note that this procedure anchors the new positions in a counterclockwise sense around $\mathrm{V}_{1}$ and $\mathrm{V}_{2}$.

For each simulation, the observed potentials of each lead system were taken as data matrices $\boldsymbol{\Phi}_{\mathrm{ECG}}(9 \times N)$ and $\boldsymbol{\Phi}_{\mathrm{ACG}}(9 \times N)$, respectively, as subsets of the total body surface potential observation $\boldsymbol{\Phi}$. In order to suppress the discretization noise, the signals were filtered with an averaging filter of length 20, having its first cutoff frequency at $50 \mathrm{~Hz}$. A column-wise zero-mean operation was applied to the matrices, forming an implicit ground for the instantaneous potentials.

\section{Singular Value Decomposition}

For all simulations and both lead systems, the singular values of these data matrices were computed by means of the Singular Value Decomposition (SVD). In the formulation for a real matrix

$$
\mathbf{\Phi}=\mathbf{U D V}^{T},
$$

the singular values, i.e., the diagonal elements of $\mathbf{D}, d_{i, i}$, were normalized with respect to the largest one. Their distributions are presented as the mean and standard deviation taken on the eight sequences for each lead system, and examined with a paired t-test.

\section{Entropy of singular value spectra}

To measure the characteristics of the singular values, the entropy of their distribution was calculated. The entropy of a decomposed data set can be formulated from the energy spectrum of its eigenvalues [8]. The entropy $H$ can be described as

$$
H(\Phi)=-\frac{1}{\ln L} \sum_{k=1}^{L} E_{k} \ln E_{k},
$$

where $\ln L$ is a normalization factor with the number of eigenvalues $L$, and

$$
E_{k}=\frac{\lambda_{k}}{\sum_{j=1}^{L} \lambda_{j}} .
$$

Using the squared singular values as $\lambda_{k}$, this measure determines the flatness of the eigenvalue spectrum. It is equivalent to measuring the energy distribution among the modes of the empirical eigenfunctions. A zero entropy indicates that only one component, $E_{1}=1$, is needed to reproduce the entire dynamics, and $H=1$ when the energy is spread out evenly across all modes.

The dispersion of the singular values also provides an indication of the complexity of the signal dynamics. That is, in the case where only $n$ singular values are significant and the others negligible, the dynamics of the signals evolve in a hyperplane of dimension $n$. Therefore, the signals have a simpler dynamics when fewer singular values are dominant.

\section{Results}

The simulated AF signals exhibited wave forms with realistic amplitudes and frequencies [1].

\section{SVD}

The mean singular values of the eight simulations and their standard deviations are presented in Figure 2 for the two lead systems. There is a transition between the first values and the last ones. The first four singular values share more or less the same range in the ECG and ACG, while for the last four, the values for the ACG are clearly higher than those for the ECG. The paired t-test applied to each of those four values showed a p-value smaller than 0.01 . The standard deviations for these values are larger in the ACG, indicating a larger variation of the last singular values for the proposed lead system. The statistics of the energy sum of the last four values, i.e., $\sum_{k=5}^{8} E_{k}$, is presented in the last column of Table 1 .

\section{Entropy}

The entropies for the two leads are shown in the first column of Table 1. The entropy value for the ACG is $8 \%$ larger than that for the ECG. 


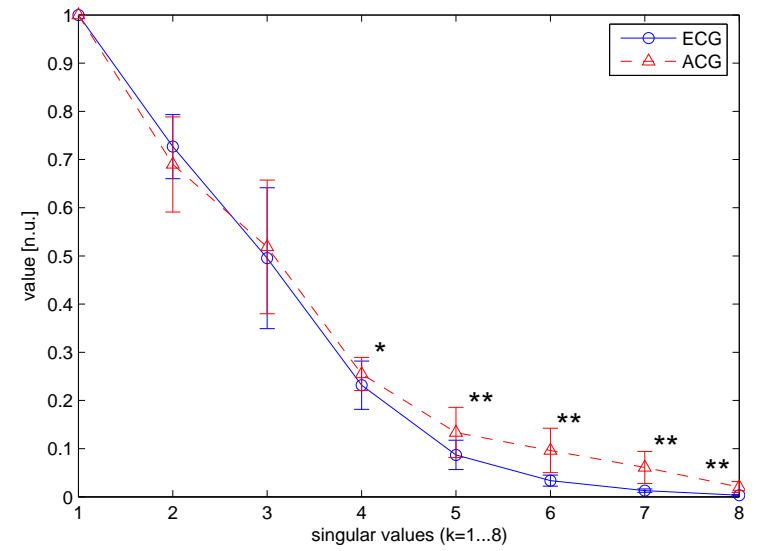

Figure 2. The normalized singular values averaged among eight different simulation data. The error bars indicate the standard deviations. The asterisks refer to the $p$-values of the paired t-test (ECG vs ACG); *: $p<0.05$, $* *: p<0.01$.

Table 1. Statistics of the entropy and partial sum of energy measure of the two lead systems. The values are presented in (mean $\pm S D)$. The asterisks indicate the p-value of the paired t-test (ECG vs ACG); *: $p<0.05$, **: $p<0.01$.

\begin{tabular}{ccc}
\hline \hline Lead System & Entropy & $\sum_{k=5}^{8} E_{k}$ [n.u.] \\
\hline ECG & $0.51 \pm 0.07$ & $0.005 \pm 0.003$ \\
ACG & $0.55 \pm 0.06 *$ & $0.018 \pm 0.011 * *$ \\
\hline \hline
\end{tabular}

\section{Discussion and conclusions}

The normalized singular value spectra with consistent larger values, especially for the last ones, suggest that the signals observed on the ACG lead system carry a higher complexity. The assessment of the energy spectrum entropy leads to the same argument, i.e., the energy of the signal dynamics is spread across more modes of the data.

The behavior of the last values does not correspond to that of white noise, for which the singular values should come up with a stable and constant value at the noise level.

The biophysical model allowed us to deal with a pure set of signals, in this case the atrial activity, uncontaminated by any extrinsic interferences, in particular that of the involvement of ventricular activity. The simulated AF sequences have realistic wave forms and behavior. The spectrum of the singular values of the body surface potential data suggests that the ACG lead systems catches more information of the atrial activity than the standard 12-lead ECG. The implication of this is that the positions of the nine electrodes used in a standard ECG recording are not optimal for retrieving the atrial content of the surface potentials.
The proposed ACG leads are a heuristic alternative to the standard 12-leads, and an easy-to-use way of measuring the body surface potentials with a focus on the atrial activity. This observation must be tested by extending our study to the clinical signals. This is why we are currently using this system in a clinical evaluation on patients suffering from $\mathrm{AF}$.

Our future works are oriented to the systematic search for the optimal lead system dedicated to the extraction of atrial activity for the analysis of atrial arrhythmias.

\section{Acknowledgements}

This study was made possible by grants from the TheoRossi-Di-Montelera Foundation, Medtronic Inc., the Swiss Governmental Commission of Innovative Technologies (CTI), and the Swiss National Science Foundation (SNSF).

\section{References}

[1] van Oosterom A, Jacquemet V. Genesis of the P wave: Atrial signals as generated by the equivalent double layer source model. Europace 2005;7:S19-S29.

[2] Jacquemet V, Virag N, Ihara Z, Dang L, Blanc O, Zozor S, Vesin JM, Kappenberger L, Henriquez C. Study of unipolar electrogram morphology in a computer model of atrial fibrillation. J Cardiovasc Electrophysiol 2003;14:S172-S179.

[3] Virag N, Jacquemet V, Henriquez CS, Zozor S, Blanc O, Vesin JM, Pruvot E, Kappenberger L. Study of atrial arrhythmias in a computer model based on magnetic resonance images of human atria. Chaos 2002;12:754-763.

[4] Courtemanche M, Ramirez R, Nattel S. Ionic mechanisms underlying human atrial action potential properties: insights from a mathematical model. Am J Physiol 1998;275:H301321.

[5] Huiskamp GJM, van Oosterom A. The depolarization sequence of the human heart surface computed from measured body surface potentials. IEEE Trans Biomed Eng 1998; 35:1047-1058.

[6] van Oosterom A. Genesis of the T wave as based on an equivalent surface source model. J Electrocardiol 2001;34:217227.

[7] van Oosterom A, Oostendorp TF. ECGSIM an interactive tool for simulating QRST wave forms. Heart 2004;90:165168.

[8] Palacios A, Gunaratne GH, Gorman M, Robbins KA. Karhunen-Loève analysis of spatiotemporal flame patterns. Phys Rev E 1998;57:5958-5971.

Address for correspondence:

Zenichi Ihara

EPFL-STI-ITS-LTS1

ELD241, Station 11

CH-1015 Lausanne

zenichi.ihara@epfl.ch 